\title{
HERRAMIENTA DE SEGUIMIENTO FUNCIONAL Y SUPERACIÓN DE INTERFASE DURANTE LA REEDUCACIÓN DEL FUTBOLISTA LESIONADO.
}

\section{A TOOL FOR FUNCTIONAL FOLLOWING AND IMPROVEMENT INTERPHASE DURING THE RETRAINING OF INJURED FOOTBALL`S PLAYER.}

\author{
Gómez Piqueras, P. ${ }^{1}$, Ortega Jimenez, J.M. ${ }^{1}$, Sainz de Baranda Andújar, P. ${ }^{2}$ \\ pedrogomez7@hotmail.com \\ 1 Universidad Castilla la Mancha, España. \\ ${ }_{1}^{1}$ Universidad de Murcia. Campus de Excelencia Mare Nostrum, España. \\ Recibido: Junio/2013 - Aceptado: Noviembre/2013.
}

\begin{abstract}
Resumen
Conscientes de la importancia de una vuelta segura del futbolista lesionado, y sabedores del complejo proceso de toma de decisión respecto al momento de la vuelta al entrenamiento grupal, presentamos una herramienta de trabajo y registro que a la vez que nos permita controlar el seguimiento y la evolución de nuestro deportista, complemente al resto de pruebas objetivas realizadas (condicionales, funcionales, psicológicas..) colaborando en la determinación del momento idóneo de retorno.
\end{abstract}

Palabras clave: Lesión, Vuelta al entrenamiento, Readaptación, Progresión funcional, Fútbol.

\begin{abstract}
Conscious about the importance of a sure injured footballer's return to play, and knowing the complex process of decision relative to the ideal moment for the return in group training, we present a tool of work and register that while permitting control us the follow-up and the evolution of our sportsman, complement to the objectives tests remainders carried out (conditional, functional, psychological..) collaborating in the determination of the suitable moment of return.
\end{abstract}

Keywords: Body Composition, Physical Activity, Physical Education, Childhood Education.

\section{INTRODUCCIÓN}

Dos de los documentos de consenso básicos en la bibliografía referente a lesiones deportivas en fútbol definen la lesión respectivamente como "cualquier daño físico sufrido por un jugador como resultado de un partido o un entrenamiento de fútbol, con independencia de la necesidad de atención médica o el tiempo de ausencia de actividades de fútbol" (Fuller et al., 2006), y "aquella circunstancia que ocurre durante una sesión de entrenamiento o partido programados que causa ausencia del jugador en el próximo entrenamiento o partido" (Ekstrand \& Gillquist, 1983)

Además de diferentes definiciones, al consultar la bibliografía especializada se proponen varias y muy distintas clasificaciones desde el punto de vista de la severidad de la lesión, siendo la más comúnmente aceptada para los trabajos que tratan la epidemiología en el fútbol la citada por San Román (2010) en base a diferentes trabajos ("Fuller et al., 2006; Hägglund, Walden, Bahr \& Ekstrand, 2005; Hägglund, Walden \& Ekstrand, 2006; Woods, Hawkins, Hulse \& Hodson, 2002”):

- Lesiones sin ausencia: no ocasionan ningún día de ausencia.

- Lesiones leves: de 1 a 3 días de ausencia de la práctica deportiva plena.

- Lesiones menores: ausencia de la práctica deportiva de 4 a 7 días.

- Lesiones moderadas: ausencia de la práctica a partir de 8 días y hasta 28.

- Lesiones graves: ausencia de la práctica deportiva durante más de 28 días.
- Lesiones final de carrera: ocasionan el final de la carrera futbolística.

En relación a su potencial de aparición, son numerosos y muy variados los trabajos que afirman el alto riesgo de sufrir una lesión deportiva que tienen los jugadores de fútbol y la gran incidencia lesiva de éstos en comparación tanto con otras actividades deportivas como con otro tipo de actividades profesionales (Llana, Pérez y Lledó, 2009). Estos trabajos, además, justifican la importancia de este tema, sobre todo en aquellos deportes como el fútbol con grandes inversiones e intereses económicos implicados donde las lesiones suponen un gran perjuicio económico en el momento en que condicionan el rendimiento y la consecución de los objetivos deportivos de jugadores y clubes ("Casáis, 2008; Cos, Cos, Buenaventura, Pruna y Ekstrand, 2010; Dvorak \& Junge, 2000; Junge, Dvorak \& GrafBaumann,2004; Rodas, Til, Medina, Solé y Ardévol, 2006; Wong \& Hong, 2005”).

En concreto, Cos et al. (2010) hacen una cita que ayuda a hacerse una idea de la importancia del problema de las lesiones en el fútbol de alto nivel (Ekstrand et al., 2002), allí se comenta que se producen 9 lesiones por cada 1.000 horas de juego (30 lesiones por cada 1.000 horas de competición y 4 por cada 1.000 horas de entrenamiento (Rodas et al., 2006), y se compara este dato con los de una empresa convencional de 25 trabajadores en la que esto supondría tener a 9 trabajadores de baja laboral por lesión cada semana. El mismo autor, citando como referencia el modelo de la UEFA de Hägglund et al (2005) señalan que a pesar de la ya comentada disparidad en los resultados de diferentes estudios, en todos ellos el riesgo lesional es alto, con una incidencia de entre un 65 a $91 \%$ de 
jugadores lesionados a lo largo de una temporada. Los datos extraídos de un estudio epidemiológico desarrollado por la UEFA en la temporada 2003-2004 con 12 equipos de primer nivel europeos (entre los que estaba el FC Barcelona) el número medio de días de baja por lesión fue de 13 días ( 2 partidos y 8 sesiones de entrenamiento perdidas) Rodas et al., (2006).

En consecuencia de la importancia expuesta, y aunque las lesiones son inherentes a la práctica deportiva, su origen multifactorial (Meeuwise, Tyreman, Hagel \& Emery, 2007) obliga a los distintos profesionales relacionados con esta a desarrollar protocolos de prevención y readaptación con un enfoque también multidisciplinario (Cos et al., 2010), que no se limiten a la monocausalidad lesiva, y aborden el proceso desde los diferentes focos interactivos del proceso (condicional, funcional, psicológico...).

\section{REEDUCACIÓN FUNCIONAL DEPORTIVA FRENTE A RIESGO DE RECIDIVA}

En el trabajo de Cos et al., (2010) citando el documento del "UEFA Model" (Hägglund et al, 2005) se dice que un futbolista se considera plenamente recuperado de una lesión cuando éste "participa al $100 \%$ de las sesiones de entrenamiento y está en disposición de disputar partidos. Si el jugador participa sólo en parte de los entrenamientos o estos son modificados y/o adaptados, el jugador no será considerado aún rehabilitado”.

Las recidivas se definen como "una lesión del mismo tipo y en la misma localización que una anterior y que sucede después del regreso de un jugador a la participación total desde la lesión inicial" (Fuller et al., 2006). En el mismo trabajo se cita que si esta recaída sucede en los dos meses posteriores al regreso a la participación total se denominará "recidiva temprana", entre los 2 y los 12 meses posteriores al regreso será "recidiva tardía", y "recidiva retrasada" si ocurre después de los 12 meses del regreso a la actividad total. Romero y Tous en 2010, citando a Drawer \& Fuller (2002) indican que las recidivas suelen ser más severas que las lesiones originales, concretamente provocando un periodo de ausencia 33\% mayor. Las recidivas se dan entre un 24 y un $30 \%$ de casos en el estudio de Hägglund et al. (2005).

Siendo conscientes por tanto de que una mala rehabilitación y una temprana vuelta a la práctica deportiva, junto al historial lesivo previo, se presentan como principales causas de la a aparición de un proceso lesivo recidivante ("Chomiak, Astrid, Peterson \& Dvorak, 2000; Ekstrand \& Gillquist,1983”), se presenta el proceso de Reeducación Funcional Deportiva (RFD) como principal herramienta para hacer frente a esta casuística.

Siguiendo a Lalín y Peirau (2011), la RFD atendería al proceso enmarcado desde el inicio de la lesión deportiva hasta la vuelta a la práctica del lesionado, que establece además programas preventivos que ayudan a evitar recaídas y anticiparse a la aparición del accidente deportivo. Dicho proceso intenta aunar el concepto médico y el de rendimiento, y para ello, se sirve del marco educativo como nexo de unión entre ambos, tratando de reeducar gestos y habilidades que el jugador realizaba con normalidad antes de la lesión, así como la base condicional sobre la que estos se desarrollaban y que en toda lesión se ve alterada por el proceso de desentrenamiento. Así, anticiparse a la aparición de la lesión y en el caso de que esto suceda favorecer la pronta vuelta a la práctica deportiva en las mismas condiciones que estaba antes de lesionarse, serán los principales objetivos perseguidos por esta actuación multidisciplinar (Lalín y Peirau ,2011).

Para facilitar la comprensión del mismo y con el propósito de establecer las bases de un lenguaje común entre los responsables de esta área, Lalín y Peirau (2011) estructuran el proceso en tres fases claramente diferenciadas: Recuperación, Readaptación y Reentrenamiento (véase figura 1).

Fig1- Estructuración y Secuenciación del programa reeducativo: Elaboración propia en base a: “Kisner \& Colby ,2002; Lalín y Peirau, 2011; Lago, 2002; Paredes ,2009”. Imágenes software Grafut.

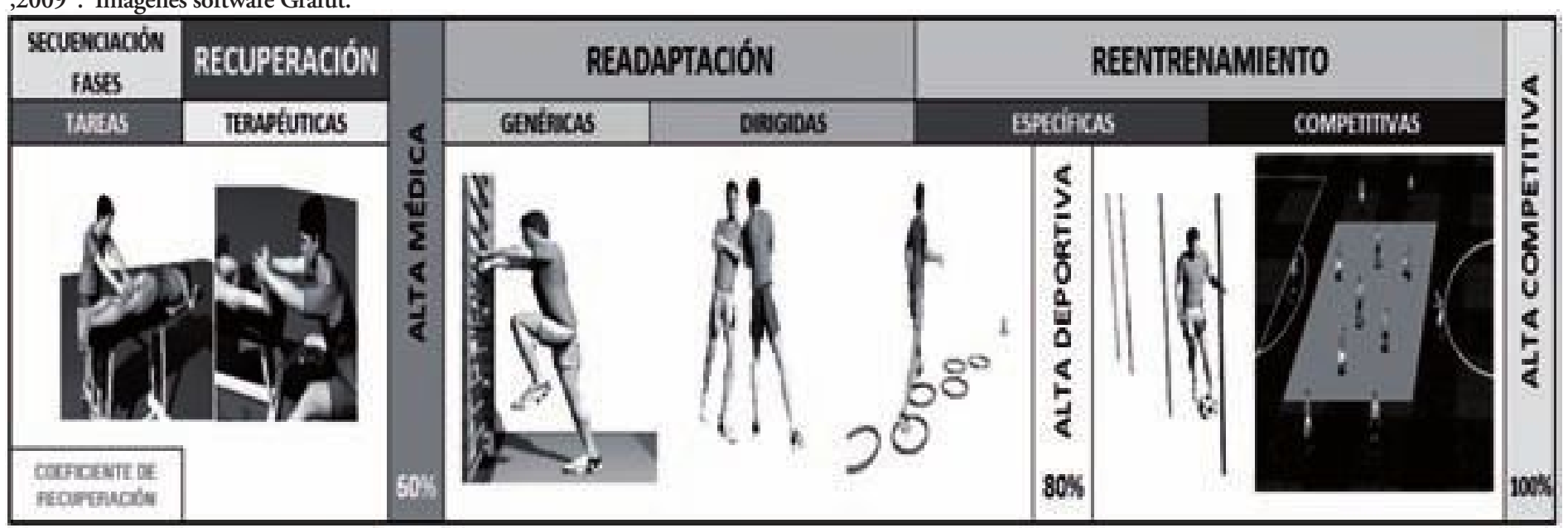


Durante la primera etapa de RECUPERACIÓN, se respetará y permitirá en la medida de lo posible favorecer que los procesos reparadores de nuestro organismo actúen adecuadamente (Lalín y Peirau, 2011), pudiendo ser necesaria una fase de inmovilización previa supeditada en tiempo y forma al diagnóstico concreto de la lesión.

En la READAPTACIÓN, etapa más funcional, el jugador deberá readquirir las habilidades básicas que afecten a la zona lesionada (correr, saltar...) a un nivel de exigencia comparable al previo de la lesión.

De modo que será en el REENTRENAMIENTO donde se deberán alcanzar los gestos específicos avanzados e integrados en la colectividad de la modalidad deportiva en cuestión.

El objetivo de la primera etapa, la de Recuperación tendrá una orientación más clínica consiguiendo una vez superada el alta médica. En las etapas de Readaptación y Reentrenamiento se debe alcanzar un alta deportiva (que no competitiva) que capacite al jugador para volver a entrenar en las mismas condiciones que el resto de compañeros no lesionados, alcanzando en el último momento el alta competitiva.

\section{LAS PROGRESIONES FUNCIONALES Y SU IMPORTANCIA EN EL RETURN TO PLAY}

La Progresión funcional, definida por McGee en 1999, consistiría en la sucesión de actividades que estimulen las capacidades motoras y deportivas del deportista, permitiendo a su vez que este adquiera y recupere la capacidades necesarias para llevar a cabo esfuerzos deportivos con seguridad y eficacia.

La utilización de medios de entrenamiento (condicionales, funcionales y específicos) de manera progresiva y funcional tendrá como principal propósito la recuperación rápida y segura del nivel óptimo del futbolista antes de volver al entrenamiento grupal y poder competir, para lo cual, a tenor de lo expuesto por Domínguez, (2012) y Kibler \& Chandler , (1994), se torna indispensable que éstos, antes de diseñarse, tengan en cuenta factores como el tipo de modalidad deportiva, el mapa condicional y muscular de la actividad, la demarcación del jugador, el momento de la temporada, la tolerancia del jugador y su respuesta psicológica.

Ya en 1994, Kibler \& Chandler aseveraban que el deportista lesionado, antes de recibir el alta deportiva, debería de haber completado progresiones funcionales que implicasen tareas propias de la especialidad deportiva (lanzar, pasar, conducir, regatear..)

Haber vivenciado y superado de manera adecuada, segura y sin dolor, estos gestos específicos caracterizados por un alto contenido de fútbol, no sólo aportará al futbolista beneficios desde el punto de vista condicional y coordinativo, sino que también influirá positivamente en su aspecto psicológico ("Bauman, 2005; Glazer, 2009”).
El Return to Play, definido por el consejo de Medicina Deportiva celebrado en Estados Unidos y publicado por Herring et al en 2002, se define como

"el proceso de decisión de cuando un deportista lesionado puede volver con seguridad a los entrenamientos y a la competición". Nuestra experiencia nos dice que, aun habiendo superado estas fases funcionales, permitir que el jugador se reincorpore al entrenamiento grupal y a la competición no es una decisión fácil, y los criterios deben ser utilizados con precaución y prudencia teniendo en cuenta al deportista y la situación del momento (Domínguez,2012).

La no existencia de una opinión consensuada al respecto del tiempo necesario para un retorno de modo seguro, convierten a esta, en una decisión subjetiva, donde el tiempo y los plazos para volver a jugar dependen de la gravedad y complejidad de la lesión (Hägglund et al., 2005).

Fuller et al (2006) defienden la idea de que en la mayoría de los clubes no se dispone de unos criterios concretos por los que un jugador lesionado pueda volver al grupo, y proponen que es recomendable disponer del perfil fisiológico y de los valores del estado físico del jugador cuando está sano, y de este modo poder ser utilizados como un punto de referencia.

\section{PRESENTACIÓN DE LA HERRAMIENTA DE SEGUIMIENTO FUNCIONAL Y SUPERACIÓN INTERFASE}

Si el control y seguimiento del futbolista lesionado se antoja como un aspecto clave del éxito de la recuperación funcional del jugador, serán de obligada inclusión en nuestro programa reeducativo, herramientas de control y seguimiento que nos permitan alcanzar este objetivo.

Puesto que la mayoría de publicaciones revisadas ("Creighton, Shrier, Shultz, Meeuwisse \& Matheson, 2010 ; Herring et al., 2002; McGee,1999; Nelson \& Butterwick , 1989”) aun utilizando criterios clínicos, condicionales, funcionales, psicológicos y subjetivos, no otorgan, a nuestro modo de ver, la necesaria importancia consensuada en 2002 al respecto de la recuperación de las habilidades deportivas específicas como aspecto clave antes de permitir el Return to play, consideramos que aportar una herramienta de seguimiento, que permita controlar y adecuar el progreso específico de las habilidades propias del deporte, podría ayudar a completar el "vacío" considerado al respecto.

La búsqueda bibliográfica realizada en las principales bases de datos (Pubmed, Sportdiscus y TESEO) previamente al desarrollo de nuestra propuesta no arrojó resultados satisfactorios, pues aunque fueron varios los trabajos que hacían mención a la importancia de incorporar herramientas de control para habilidades funcionales y específicas del deporte ("Herring et al., 2002; Creighton et al 2010”), tan sólo nos fueron de utilidad debido a su mayor concreción, ejemplos genéricos como el de (Fuller \& Walker,2006) 
diferenciando entre habilidades básicas con balón y habilidades de partido, y la propuesta de Rosch et al (2000) en la batería de test estandarizado F-MARC TEST, planteando 8 pruebas de habilidad con balón con el objetivo de evaluar al jugador en relación a las acciones técnicas individuales de juego.

De este modo, y como ya planteamos en Gómez, Ortega y Sainz de Baranda (2013), se hace necesaria una progresión acorde a la complejidad situacional que el jugador encontrará nada más incorporarse con el grupo, no limitándonos meramente a aspectos de tipo individual, sino integrando la realidad socio afectiva del fútbol a la progresiva red de situaciones vivenciadas por el lesionado.

El instrumento presentado, a raíz de la problemática detectada y mencionada, fue propuesto y desarrollado, con la intención de presentar una aceptable validez de contenido, por un grupo de expertos, (compuesto por 5 licenciados en Ciencias de la Actividad Física y el deporte con especialidad en Fútbol y Recuperación de lesiones, un fisioterapeuta con amplia experiencia en el ámbito del fútbol y 2 Doctores con demostrada experiencia en el ámbito investigador), en base a su conocimiento del campo de estudio y las escasas referencias similares encontradas en la literatura científica.

Esta herramienta (véase Figura 2), presentada como cuestionario a rellenar por parte del responsable/s del jugador lesionado durante los periodos de readaptación y reentrenamiento, se divide en 3 fases de contenidos, las cuales, progresivas en complejidad, se compondrán cada una de ellas de 8 items (medios de entrenamiento) valorados, a través de una sencilla escala de tipo Likert , en relación a 3 parámetros: (1.- Velocidad de ejecución, 2.- Confianza del jugador y 3.- Molestias presentadas), los cuales, aun siendo rellenados en última instancia por el observador, requerirán complementarse con la opinión del lesionado, quien deberá transmitir sus sensaciones en variables tan subjetivas como las presentadas.

Dada la sencillez de los elementos técnico tácticos evaluados, no se requiere una formación previa en el uso de la herramienta para poder utilizar la misma, pues todo profesional con conocimientos básicos sobre los elementos valorados podrá valorar la correcta ejecución de los mismos. Aun con todo, sería preferible que dichas valoraciones fuesen realizadas por un experto en motricidad y/o recuperación.

La puntuación total obtenida en cada una de las fases, correlacionada de manera visual con el funcionamiento de un semáforo (Verde=Apto, Rojo= Prohibido, Amarillo= Depende), nos ayudará a decidir si el jugador puede seguir progresando en contenidos y medios de entrenamiento específicos, o si por el contrario, debe mantenerse en la misma fase por no haber sido capaz de superar con confianza, velocidad adecuada y ausencia de dolor las habilidades futbolísticas valoradas.
Debido a la dependencia que la herramienta presenta con la opinión del observador, se recomienda que el seguimiento a un lesionado sea realizado siempre por el mismo profesional, de modo que la información arrojada sea lo más objetiva y fiable posible.

Puesto que los medios de entrenamiento específicos se irán añadiendo progresivamente en base a las sensaciones del jugador, esta herramienta no sólo nos será útil como información complementaria en la decisión del Return to Play, sino que servirá de referencia y guía para la incorporación de situaciones con mayor exigencia y solicitación para la estructura dañada, respetando, como es lógico, la individualidad y particularidad de cada tipo de lesión.

\section{LIMITACIONES DE LA PROPUESTA}

Consideramos de extrema importancia subrayar que lo aquí presentado se limita a una propuesta contextualizada a nuestro entorno y llevada a cabo por nuestro grupo de trabajo durante las últimas dos temporadas. Esta herramienta, sin tener una validez y fiabilidad científicamente demostradas, ha arrojado información muy útil durante el proceso de readaptación de nuestros lesionados, razón por la cual, consideramos interesante compartirla.

Dicho esto, y convencidos de que la escasa existencia de herramientas similares en la literatura científica condiciona el desarrollo y validación de la presente, animamos a seguir profundizando en el desarrollo de instrumentos que den solución a la problemática presentada, pues consideramos que las limitaciones encontradas en lo propuesto por nuestro grupo de trabajo, podrían suponer interesantes puntos de partida en futuras investigaciones.

De este modo:

- Convendría aumentar la validez de contenido reuniendo a un número mayor de expertos colaboradores en el desarrollo del cuestionario, o bien, apoyándose en herramientas ya validadas y tal vez, no tenidas en cuenta en el presente.

- Un análisis de validez concurrente, comparando los valores alcanzados en esta herramienta con mejoras condicionales o funcionales paralelas supondría un refuerzo más que interesante para nuestra propuesta.

- Puesto que las connotaciones inherentes a cada uno de los distintos tipos de lesión (articular, muscular, ósea) son diferentes, se debería valorar la posibilidad de establecer distintas escalas de valoración en función de estos.

- La dependencia del observador debería de ser minimizada, de forma que la herramienta garantizase medir siempre lo mismo independientemente de quien lo valorase.

- La subjetividad de parámetros tan importantes para nosotros como la confianza y molestia del 
deportista no tienen cabida en metodologías de tipo cuantitativo, debiéndose a nuestro parecer, ampliar y complementar las valoraciones con métodos más cualitativos.

\section{CONCLUSIÓN}

Convencidos de la anteriormente citada multicausalidad factorial en el proceso lesivo, defendemos la utilización de la herramienta presentada en el presente artículo como una ayuda complementaria en el complejo proceso de toma de decisión de vuelta al grupo, la cual, deberá apoyarse en otros criterios ya mencionados y

\section{REFERENCIAS}

Bauman, J . (2005). Returning to play: the mind does matter. Clin J Sport Med,15,432-435.

Casáis, L. (2008). Revisión de las estrategias para la prevención de lesiones en el deporte desde la actividad física. Apunts. Medicina de l'Esport, 157, 30-40.

Chomiak, J., Astrid, J., Peterson, L \& Dvorak, J.(2000). Severe injuries in football players. Influencing factors. Am J Sports Med,28(5 Suppl),58-68.

Cos, F., Cos, M.A., Buenaventura, L., Pruna,R y Ekstrand,J. (2010). Modelos de análisis para la prevención de lesiones en el deporte. Estudio epidemiológico de lesiones: el modelo Union of European Football Associations en el fútbol. Apunts. Medicina de l'Esport, 45 (166), 95-102.

Creighton, D., Shrier, I., Shultz,R., Meeuwisse, W \& Matheson,G. (2010). Return to play in sport: A decision-based Model. Clin J Sport Med, 20, 5.

Dominguez,E. (2012). Material Docente del Módulo de "Readaptación" del Máster Universitario de Prevención y Readaptación Físico-Deportiva de Lesiones en el Fútbol. Universidad de Castilla La Mancha. RFEF.

Drawer, S \& Fuller, C.W. (2002). Evaluating the level of injury in English professional football. Br J Sports Med, 36,446-451.

Dvorak, J \& Junge, A. (2000). Football injuries and physical symtoms: a review of the literature. Am J Sports Med, 28(5), S3-S9.

Ekstrand, J \& Gillquist, J. (1983). Soccer injuries and their mechanism: a prospective study. Med Sci Sports, 15(3),267-270.

Fuller, C.W., Ekstrand, J., Junge,A., Andersen, T., Bahr,R., Dvorak,J., ... Meeuwisse,W. (2006). Consensus statement on injury definitions and data collection procedures in studies of football (soccer) injuries. Scand J Med Sci Sports, 16, 83-92.

Fuller,C.W \& Walker, J. (2006). Quantifying the functional rehabilitation of injured football players. $\mathrm{Br}$ J Sports Med, 40,151-157.

Glazer, DD.(2009) Development and preliminary también de imprescindible valoración y seguimiento (fuerza, flexibilidad, resistencia, salto, coordinación, ansiedad, miedo, adherencia...).

Establecer y aplicar herramientas de control en pos de la objetivización del proceso de toma de decisión respecto al retorno del futbolista lesionado, no solo oprimirá el espacio concedido a la subjetividad profesional, sino que contribuirá a su vez a dotar de mayores garantías las decisiones tomadas por el equipo de trabajo multidisciplinar, sin eliminar totalmente, debido a la naturaleza compleja del fenómeno lesivo, el indeseado riesgo de recaída.

validation of the Injury-Psychological Readiness to Return to Sport (I-PRRS) scale. J Athl Train,44,185189.

Gómez,P., Ortega,JM y Sainz de Baranda, P. (2013) A propósito del Return top play en futbolistas lesionados. Apunts. Medicina de l'Esport ,(In Press).

Hägglund, M.., Walden, M., Bahr, R \& Ekstrand, J. (2005). Methods for epidemiological study of injuries to professional football players: developing the UEFA model. Br J Sports Med, 39,340-346.

Hägglund, M., Walden,M \& Ekstrand,J (2006). Previous injury as a risk factor for injury in elite football: a prospective study over two consecutive seasons. Br J Sports Med, 40,767-772.

Herring, S., Bergfeld,J., Boyd,J., Duffey,T., Fields,K., Grana,W., ... Sallis,R. (2002). The Team Physician and Return-To-Play Issues Consensus Statement. Med Sci Sports.

Junge, A., Dvorak, J \& Graf-Baumann, T. (2004). Football injuries during the World Cup 2002. Am J Sports Med, 32, 23S-27S.

Kibler,W.B \& Chandler,T.J. (1994) Sport-specific conditioning. Am J Sports Med, 22,424-432.

Kisner, C \& Colby, L. (2007). Therapeutic Exercise. Philadelphia: F.A. Davis Company.

Lago, C. (2002). La Preparación Física en el Fútbol. Madrid: Biblioteca Nueva.

Lalín, C y Peirau, X. (2011). La reeducación funcional deportiva. En Nacleiro, F. Entrenamiento deportivo: fundamentos y aplicaciones en diferentes deportes (Capítulo 27, 419-429). Editorial Médica Panamericana. Madrid.

Llana,S., Pérez,P y Lledó,E. (2009). La epidemiología en el fútbol: una revisión sistemática. Rev Int Med Cienc Act Fis Deporte, 10, 37.

Mc Gee (1999). Progresión funcional en la Rehabilitación. En Prentice,W. Técnicas de Rehabilitación en la Medicina Deportiva. Barcelona.Paidotribo.

Meeuwisse, W.H., Tyreman, H., Hagel, B \& Emery, 
C. (2007). A dynamic model of etiology in sport injury: The recursive nature of risk and causation. Clin J Sport Med, 17, 215-219.

Nelson,D \& Butterwick,D (1989). Guidelines for Return to Activity After Injury. Can Fam Physician, $35,1637-55$

Paredes, V. (2009). Método de cuantificación en la readaptación de lesiones en fútbol. Tesis Doctoral. Universidad Autónoma de Madrid.

Rodas, G., Til, Ll., Medina, D., Solé, J y Ardèvol, J. (2006). Prevención de lesiones en el fútbol. En: Esparza, F., Fernández, T., Martínez, J.L y Guillén, P. (eds). Prevención de las Lesiones Deportivas. Asociación Murciana de Medicina del Deporte, 51-62

Romero, D y Tous, J. (2010). Prevención de lesiones en el deporte. Claves para un rendimiento deportivo óptimo. Madrid: Editorial Médica Panamericana.

Rösch,D., Hodgson, R., Peterson, L., Graf-Baumann, T., Junge,A., Chomiak, J \& Dvorak, J. (2000). Assesment and Evaluation of Football Performance. Am J Sports Med,28,29-39.

San Román, Z. (2010). Material Docente del Módulo de "Prevención" del Máster Universitario de Prevención y Readaptación Físico-Deportiva de Lesiones en el Fútbol. Universidad de Castilla La Mancha. RFEF.

Wong, P \& Hong, Y. (2005). Soccer injury in the lower extremities. Br J Sports Med, 39, 473-482.

Woods, C., Hawkins, R., Hulse, M \& Hodson, A. (2002). The Football Association Medical Research Programme: an audit of injuries in professional footballanalysis of preseason injuries. Br J Sports Med, 36, 436441.

\section{FIG 2 - PROGRESIÓN FUNCIONAL HABILIDADES ESPECÍFICAS}

Jugador:

Lesión:

Responsable:

\section{Demarcación:}

Dia 0:

\section{Periodo Valoración:}

(Considere las siguientes indicaciones antes de rellenar la planilla de cada fase).

\begin{tabular}{|c|c|c|c|c|c|c|}
\hline PARÁMETROS A VALORAR & \multicolumn{6}{|c|}{ PUNTUACOÓN } \\
\hline \multirow{2}{*}{ VELOCIDAD EJECUCIÓN } & 0 & 1 & 2 & 3 & 4 & 5 \\
\hline & Inadecuada & & & & & Adectuada \\
\hline \multirow{2}{*}{ CONFIANZA } & 0 & 1 & 2 & 3 & 4 & 5 \\
\hline & Ninguns & & & & & Total \\
\hline \multirow{2}{*}{ MOLESTIA } & 0 & 1 & 2 & 3 & 4 & 5 \\
\hline & Ninguna & & & & & Alta \\
\hline Ne Puntos necesarios Superación de Fase & $65-80$ & sí & $55-64$ & ¿? & $0-54$ & No \\
\hline
\end{tabular}

\begin{tabular}{|c|c|c|c|c|c|}
\hline Ne & $\begin{array}{l}\text { FASE } 1 \text { : CONTEN } \\
\text { MEDIOS ENTRENAMIENTO }\end{array}$ & $\begin{array}{l}\text { BÁsICOS C } \\
\text { Velocidad }\end{array}$ & $\begin{array}{l}\text { SIN BALON } \\
\text { Confianza }\end{array}$ & Molestia & PUNTOS \\
\hline 1 & Desplazamientos posicionales sin balón & & & & \\
\hline 2 & Habilidad y Manejo del balón & & & & \\
\hline 3 & Desplazamientos con balón & & & & \\
\hline 4 & Recepciones sin oposición & & & & \\
\hline 5 & Golpeos superficies elementales & & & & \\
\hline 6 & Golpeos orientados a precisión & & & & \\
\hline 7 & $1 \times 1$ pasivo & & & & \\
\hline 8 & Asociaciones grupales básicas & & & & \\
\hline & & & & L PUNTOS & 0 \\
\hline
\end{tabular}




\begin{tabular}{|c|c|c|c|c|c|}
\hline \multicolumn{7}{|c|}{ FASE 2: CONTENIDOS AVANZADOS CON BALÓN } \\
\hline N2 & MEDIOS ENTRENAMIENTO & Velocidad & Confianza & Molestia & PUNTOS \\
\hline $\mathbf{1}$ & Conducción con oposición & & & & \\
\hline $\mathbf{2}$ & Recepciones con incertidumbre ó/y oposición & & & & \\
\hline $\mathbf{3}$ & Golpeos orientados a potencia & & & & \\
\hline $\mathbf{4}$ & Figura tècnica grupal & & & & \\
\hline $\mathbf{5}$ & Especificas ofensivas semicontroladas & & & & \\
\hline $\mathbf{6}$ & Especificas defensivas semicontroladas & & & & \\
\hline $\mathbf{7}$ & Secuencias grupales facilitadas & & & & \\
\hline $\mathbf{8}$ & Gesto de mayor dolor inicial controlado & & & & \\
\hline \multicolumn{7}{|c|}{} & & & TOTAL PUNTOS & \\
\hline
\end{tabular}

\begin{tabular}{|c|c|c|c|c|c|}
\hline \multicolumn{7}{|c|}{ FASE 3: CONTENIDOS GRUPALES DEL JUEGO } \\
\hline N2 & MEDIOS ENTRENAMIENTO & Velocidad & Confianza & Molestia & PUNTOS \\
\hline 1 & J.Posesión y Rondos (Apoyo exterior) & & & & \\
\hline 2 & Situaciones tácticas parciales & & & & \\
\hline 3 & Rondos dinámicos (comodin) & & & & \\
\hline 4 & Juegos de posesión reducidos (Comodín) & & & & \\
\hline 5 & Situaciones amplitud en especificidad posicional & & & & \\
\hline 6 & Situación competitiva real (comodin) & & & & \\
\hline 7 & Partido de entrenamiento & & & & \\
\hline 8 & Gesto de mayor dolor inicial real & & & & \\
\hline \multicolumn{7}{|c|}{} & & & TOTAL PUNTOS & \\
\hline
\end{tabular}

